\title{
A Study of the Effects of Cancer Patient Children on Their Healthy Siblings
}

\author{
Laura M. Barlay, Bernadette Péley \\ University of Pécs, Pécs, Hungary
}

\begin{abstract}
The objective of this paper is to explore in what ways children are affected by the presence of their cancer patient siblings in the family. The participants were 25 healthy children, whose siblings were suffering from malignant tumors, and 25 healthy children with no current illness (as a control group). Data included self- and parent- report questionnaires, KFD (Kinetic Family Drawings) and interviews. The result indicated that behavioral and attention problems have increased in the target group since the diagnosis. The scales showed significant differences on the "Withdrawn" and "Anxious" as well as on the "Internalizing” and the "Total Problems" tests. Regarding the KFD, reaction time increased in the target group and they used smaller proportions of the paper, their own figure was usually smaller and the last one in the row. Furthermore, they used fewer and fainter colours than the children in the control group. The drawings have been analyzed only in forms of patterns. Our study aimed to emphasize the vulnerability of the healthy siblings of cancer patient children due to the changed family homeostasis. In addition, the study partly proved that the KFD is a useful tool to present a subtle picture of the inner conflicts of these children, the overturned homeostasis, the changed family dynamics and emotional stresses.
\end{abstract}

Keywords: pediatric cancer, sibling relationships, drawing, pattern

\section{Introduction}

According to the assumption of the family systems theory approach, all the members of a system have an impact on the others; all the changes in the life of a family member influence all the other members (although not to an equal extent) and induce change for the whole system. A tumor diagnosed in childhood is an event of life turning over the whole homeostasis of the family. Our research dealt with the impact on the healthy brothers and sisters of cancer children. The brothers and sisters have to adapt themselves to the altered situation and they quickly face the fact that parents' attentions, which were more or less evenly divided among them earlier, focus on the sick brother or sister in the new situation and the healthy child is getting out to the periphery of attention. The usual everyday scenario is overturned and replaced by uncertainty, incalculableness and anxiety.

The fear and anxiety of the parents for their sick child have an impact on their healthy children, too: They are also concerned for their brother or sister, at the same time, they begin to worry about their own health, too (Houtzager, Grootehuis, \& Last, 2001). Several studies dealt with the consequences of the altered communication between parents and their healthy child (Bendor, 1990; Havermans \& Eiser, 1994; Alderfer,

Laura M. Barlay, Ph.D. candidate, Institute of Psychology, University of Pécs.

Bernadette Péley, Ph.D., professor, Institute of Psychology, University of Pécs. 
Laby, \& Kazak, 2003; Houtzager, Grootenhuisa, Caron, \& Lasta, 2004). The lack of open and sincere communication at the cognitive and emotional level of a healthy child may increase the above-mentioned sense of fear. Seeing the overload on their parents, healthy children often deliberately hide their own emotions, on the one hand, protecting themselves, on the other hand, sparing their parents. At the same time, there are studies emphasizing the sense of guilt, jealousy and anger of healthy children as a primary reaction to the altered situation (Labay \& Walco, 2004; Woodgate, 2006).

\section{Methods}

\section{Hypothesis}

Hypothesis 1: A malign tumor diagnosed for the brother/sister and the long-lasting chemotherapy, as well as radiotherapy afterwards, the altered image of the brother/sister, the inaccessibility of the mother for the healthy child, the uncertainty or possible failure of healing predispose the healthy child to develop emotional, behavior and concentration problems. Therefore, we expected that-in comparison to a control group, children who had no problems earlier have a higher probability to show behavior and attitude problems, causing symptoms of internalization, as well as externalization.

Hypothesis 2: The presence of a serious illness, sometimes threatening the life of a child, is not a normative event in the life of a child and of his/her brother/sister. It is a period full of stress and changes for the healthy children, too, requiring a lot of adaptation. We assumed that the problems, tensions and conflicts taking place in relation and as a consequence of the brother's/sister's illness leave a trace on the family drawings of healthy children.

\section{Participants}

Target group: the healthy brothers and sisters of 25 children suffering from a malign tumor and receiving medical treatment. Control group: the healthy brothers and sisters of 25 healthy children. The control was adjusted to the target group in terms of gender $\left(\chi_{(1, \mathrm{~N}=50)}^{2}=0.08 ; P>0.5\right)$, age $\left(t_{(48)}=0.45 ; P>0.5\right)$, number of brothers/sisters $\left(t_{(48)}=-1.22 ; P>0.5\right)$ and age difference between brothers/sisters $\left(t_{(48)}=0.89 ; P>0.5\right)$. There is no significant difference between the two groups along these variables.

\section{Testing Technique}

(1) CBCL (Child Behavior Checklist) (Achenbach, 1991). We were working with the version of the questionnaire entitled YSR (Youth Self-Report) and PRF (Parent Form).

(2) KFD (Kinetic Family Drawing) (Burns \& Kaufman, 1972). Instruction: "Draw a picture of everyone in your family, including you, doing something”. We examined the following variables of the drawings: reaction time, rate of covering the paper surface (square millimeter and in \%), the size of their own figure in millimeter, the average size of human figures in millimeter, the size of human figures in comparison to their own figure in \%, drawing sequence (figure drawn first/last), separation/encapsulation (figures separated by a line or an object), leaving out their own figure, interaction (brother/sister-mother/own figure-mother), nature of activity (mother/own figure), dynamics (encoded by two independent encoders, the values of the variables: passive, moderate and energetic), number of auxiliary elements (e.g., game, sun, cloud, car and furniture), number of colours and intensity of colours (in RGB (red-green-blue) system).

\section{Results}

In case of CBCL YSR, there is a significant difference between the target group and the control group in 
the scales of "withdrawn", "somatic complaints", "anxious/depressed” and "thought problems”, as well as in derived scales, such as the scale of "internalizing" and that of the "total problems" (see Table 1). The outstandingly high result on the scale of "thought problems" was surprising-This scale has a token value on the clinical sample according to the professional literature. Item 9 can be made responsible for the result we have measured: "I cannot get rid of certain thoughts" (Answers to the question "From what: thoughts about the illness of the brother/sister: 'what happens if I also get cancer”'). If this item is filtered out, there is no difference between the target group and the control group any more.

In case of CBCL PF, the scale of "thought problems" is left out from the previous scales, but the scales of "aggressive behavior" and "externalizing” are added to the series (see Table 2).

The results clearly show that the analyzed persons cannot be considered as a pathological group in terms of their behavioral, emotional and social relations, at the same time they may be potential candidates for long-lasting psychological-social trauma.

Table 1

Significant Difference Scales-CBCL YSR (Independent Samples t-Test: $t_{(48)}$ )

\begin{tabular}{lll}
\hline CBCL scales & $t_{(48)}$ & Significance (2-tailed) \\
\hline Withdrawn & 2.66 & 0.01 \\
Somatic complaints & 2.94 & 0.01 \\
Anxious/depressed & 3.48 & 0.00 \\
Thought problems & 4.03 & 0.00 \\
Internalizing & 7.33 & 0.00 \\
Total problems & 4.24 & 0.00 \\
\hline
\end{tabular}

Table 2

Significant Difference Scales-CBCL PF (Independent Samples $t$-Test: $t_{(48)}$ )

\begin{tabular}{lll}
\hline CBCL scales & $t_{(48)}$ & Significance (2-tailed) \\
\hline Withdrawn & 5.95 & 0.00 \\
Somatic complaints & 3.75 & 0.00 \\
Anxious/depressed & 2.33 & 0.02 \\
Aggressive behavior & 4.07 & 0.00 \\
Internalizing & 4.14 & 0.00 \\
Externalizing & 2.62 & 0.01 \\
Total problems & 4.19 & 0.00 \\
\hline
\end{tabular}

The time passed since the diagnosis has an impact on the following trends (two-variable linear regression): The initially high level of anxiety drops with the time passing, at the same time, the volume of externalization problems goes up, although not that much. The relationship between anxiety and the time passed since the diagnosis (see Figure 1): $R^{2}=0.46 ; F=19.56 ; P<0.001$; Anxious $=-2.92 *$ dg_time +70.38 .

The relationship between troubles of externalization and the time passed since the diagnosis (see Figure 2): $R^{2}=0.19 ; F=5.16 ; P=0.03$; Externalizing $=1.75 *$ dg_time +40.94 .

Age 12 (beginning of adolescence) is a limit in respect to the age of the healthy brother/sister: The values in the scale of the total problem are significantly higher in the target group in case of elder children $t_{(24)}=2.49$; $P=0.022)$. This difference in its trend is also shown by the control group, but its rate is lower and not significant $\left(t_{(24)}=0.33 ; P=0.74\right)$. 
Thought problems increase with the growth in age (see Figure 3): $R^{2}=0.31 ; \mathrm{F}=7.58 ; \mathrm{p}=0.01$. Thought problems $=1.38 *$ age +42.67 .

\section{CBCL YSR Anxious / Depressed}

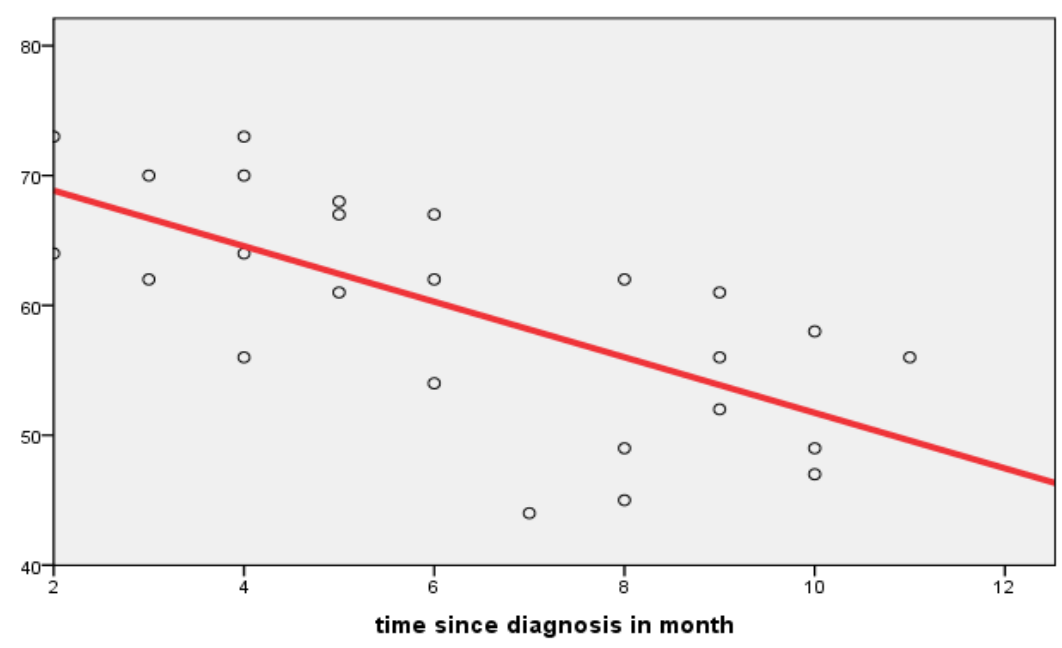

Figure 1. Scale of anxious in function of time passed since diagnosis.

CBCL YSR Externalizing

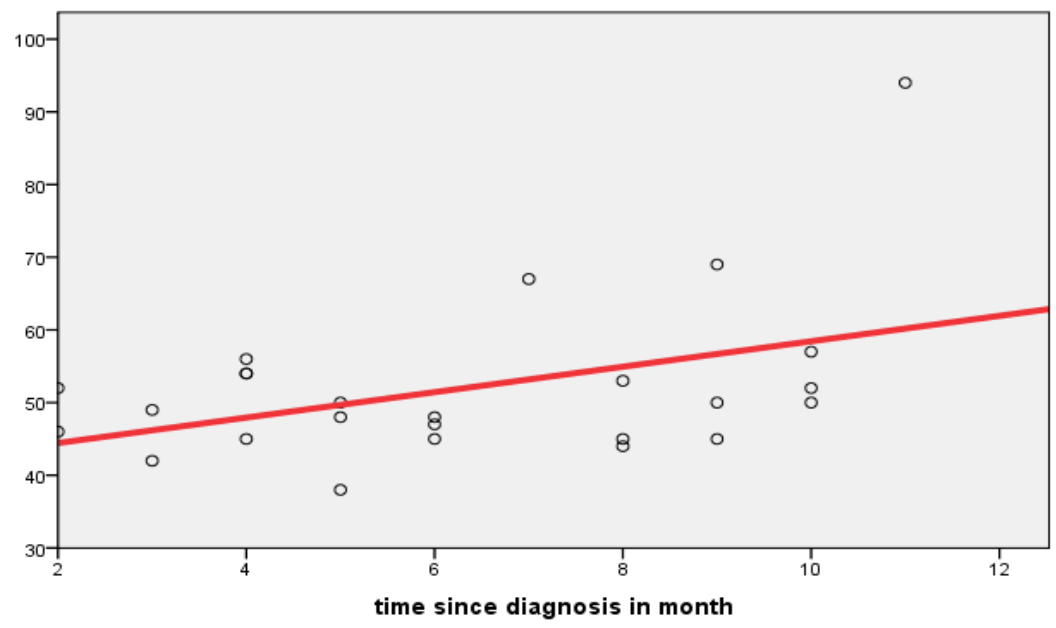

Figure 2. Scale of externalizing in function of time since diagnosis.

Figure 4 shows scale results characterizing the target group where the difference between YSR and PF is significant (the results successively are: $t_{(24)}=-2.54, P=0.02 ; t_{(24)}=3.68, P<0.001 ; t_{(24)}=-2.39, P=0.02$ ). In the control group, there is no scale where the results received from the persons (parent and child) who filled out the questionnaire are significantly different from each other.

The results showing a significant difference between the metric variables of the KFD of the target group and the control group are summarized in Table 3, the differences indicated by nominal variables (values: yes-no) are shown in Table 4.

The family drawings of healthy brothers and sisters of tumors children are more moderate in terms of dynamics, compared with the control group (based on the marking of two independent encoders: Cohen’ Kappa 
= 0.93; Mann-Whitney U Test: $U=165.00 ; P<0.001)$.

\section{CBCL Thought Problems}

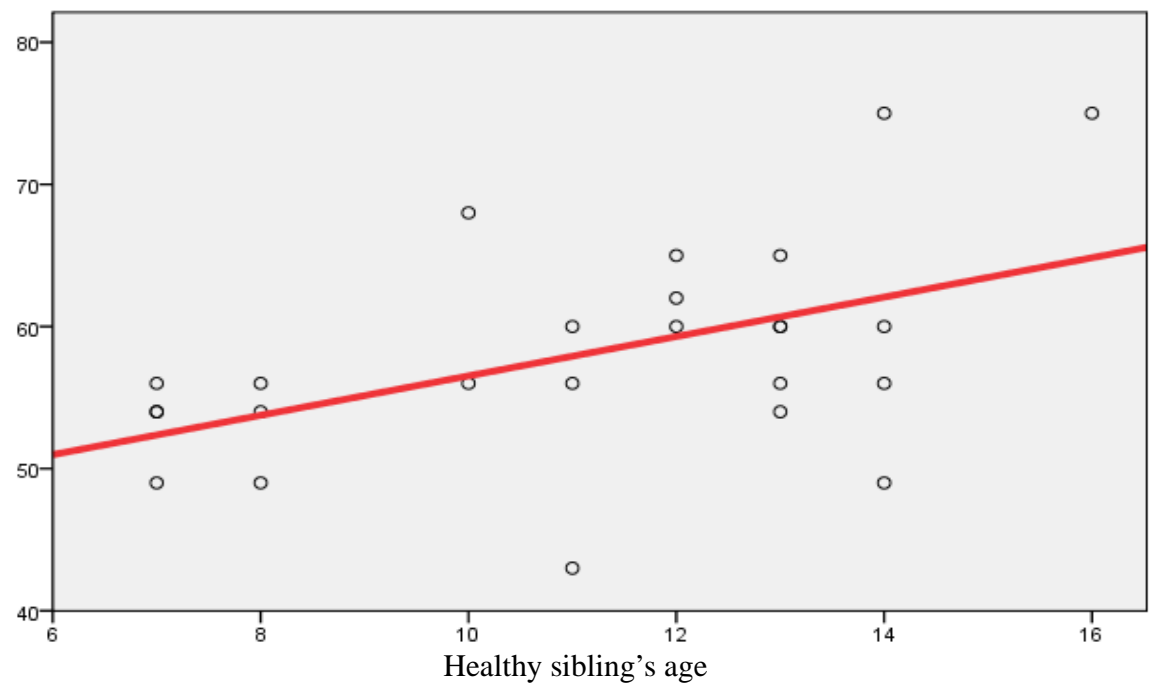

Figure 3. Scale of thought problems in function of age.

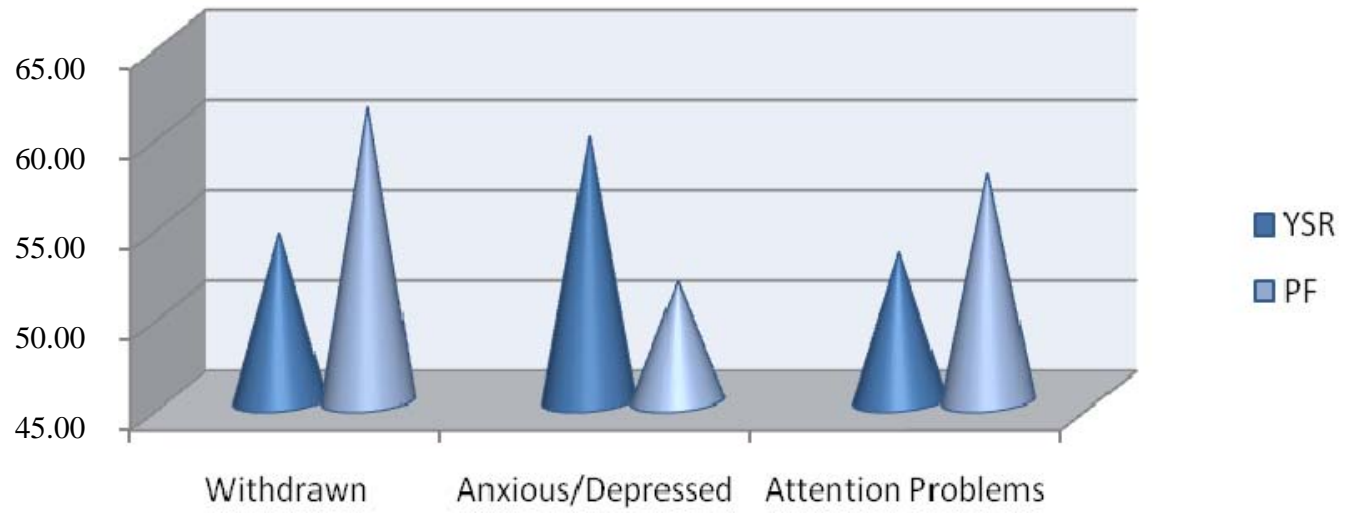

Figure 4. Scales showing a significant difference between self-characterizing and the parent's version.

Table 3

Variables of KFD Showing a Significant Difference Between the Target Group and the Control Group (Independent Samples $t$-Test: $\left.t_{(48)}\right)$ In Case of $t>0$ Target $>$ Control and in Case of $t<0$ Target $<$ Control)

\begin{tabular}{lcc}
\hline Variable name & $t_{(48)}$ & Significance (2-tailed) \\
\hline Reaction time & 5.49 & $<0.00$ \\
Rate of coverage & -3.03 & 0.00 \\
Average of figures & -3.138 & 0.003 \\
Own figure in mm & -2.372 & 0.022 \\
Size of mother & 2.723 & 0.009 \\
Size of father & 3.004 & 0.004 \\
Quantity of auxiliary elements & -3.582 & $<0.001$ \\
Intensity of colour & -4.838 & $<0.001$ \\
\hline
\end{tabular}


Table 4

Variables of KFD Showing a Significant Difference Between the Target Group and the Control Group $\left(\chi^{2}=\right.$ Pearson Chi-square Test)

\begin{tabular}{lrl}
\hline Variable name (answer: yes/no) & $\chi_{(1,50)}^{2}$ & Significance (2-tailed) \\
\hline Own figure playing & 7.58 & 0.05 \\
Brother/sister mother in interaction & 6.35 & 0.01 \\
Own figure separated & 8.42 & 0.01 \\
Own figure left out & 5.56 & 0.02 \\
Drawing own figure as the last one & 6.52 & 0.02 \\
Drawing brother/sister first & 20.78 & 0.00 \\
\hline
\end{tabular}

The drawings have been analyzed, keeping the principle of multiple causalities in view: A certain psychological phenomenon may be expressed by images in many different ways and there may be various meanings behind each graphic expression (Vass, 2001). Therefore, the phenomena of individual drawings have been analyzed only in forms of patterns. In this sense, although the drawings show an infinite variety, we have searched drawing features that often appear together, which is forming patterns and psychological phenomena can be associated with them. In this way, the different forms of appearance do not present an infinite variety any more. During our work, we have made an effort to describe the patterns in the above-mentioned sense on the family drawings of brothers/sisters of cancerous children. These configurations have been developed in comparison with an objective measurement (CBCL PF/YSR), ensuring the validation of patterns and avoiding a rightful criticism of mechanic interpretation, which explain the drawings with psychological phenomena of a questionable justification.

We will illustrate each pattern with a prototype, side by side with a drawing from the control group in contrast.

(1) Pattern 1 (see Figures 5 and 6):

Reaction time $>20$ seconds; low coverage of the paper surface $(<26 \%)$; average size of figures $<25 \mathrm{~mm}$; their own figure was left out from the drawing (it was made up after the question of the test leader); self without interaction, while the brothers/sisters are in interaction; use of eraser; high value of CBCL YSR scale of Anxious/Depressed ( $M=59.86)$; high value on CBCL YSR scale of Withdrawn $(M=61.78)$.

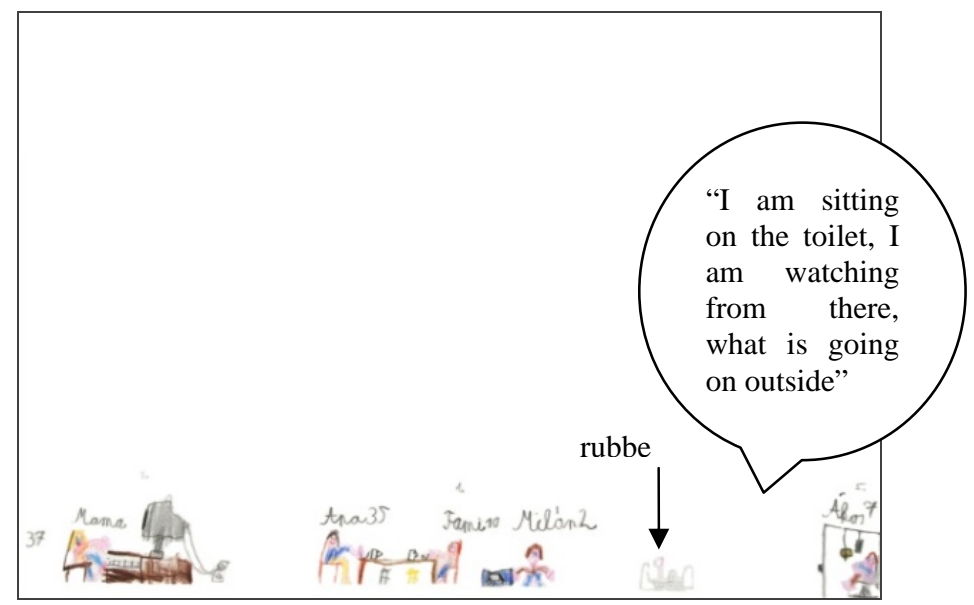

Figure 5. A 8-year-old boy, his sister suffering from leukemia. Low coverage of the paper surface. The people are small. The healthy brother is isolated from family (right side). The self is without interaction, while the siblings are in interaction. 


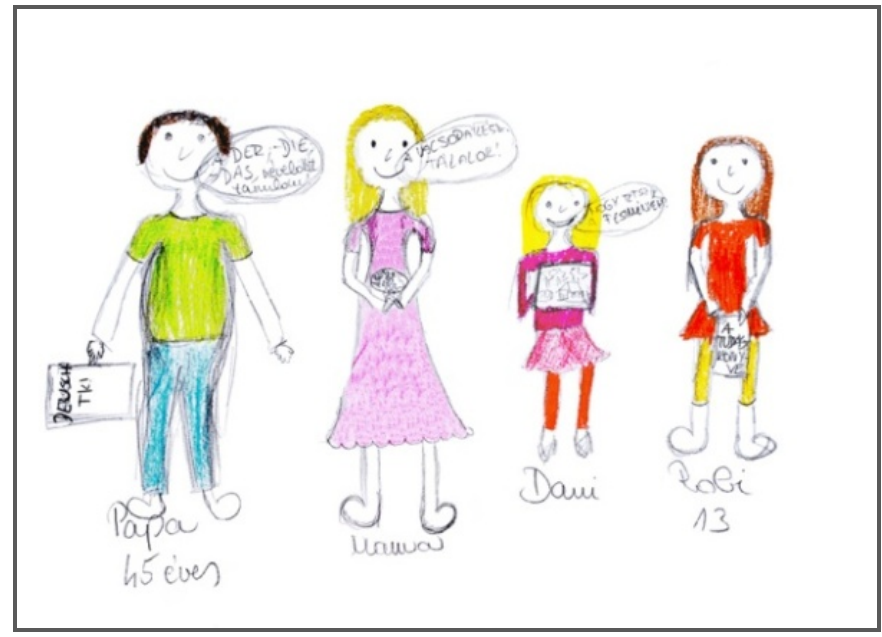

Figure 6. A 9-year-old girl, control group. High coverage of the paper surface. The family is playfully together, normal coverage of the paper surface.

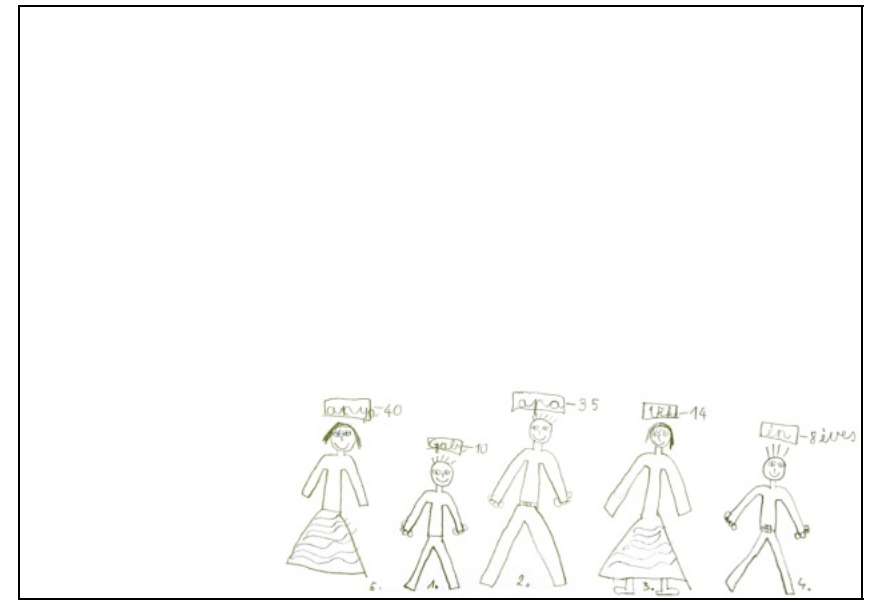

Figure 7. A 8-year-old boy, his bother suffering from leukemia. The drawer is on the right side of paper. The people have not hands or legs (essential detail of a human figure). The drawer used only one colour and there is black. The members are passive (without action).

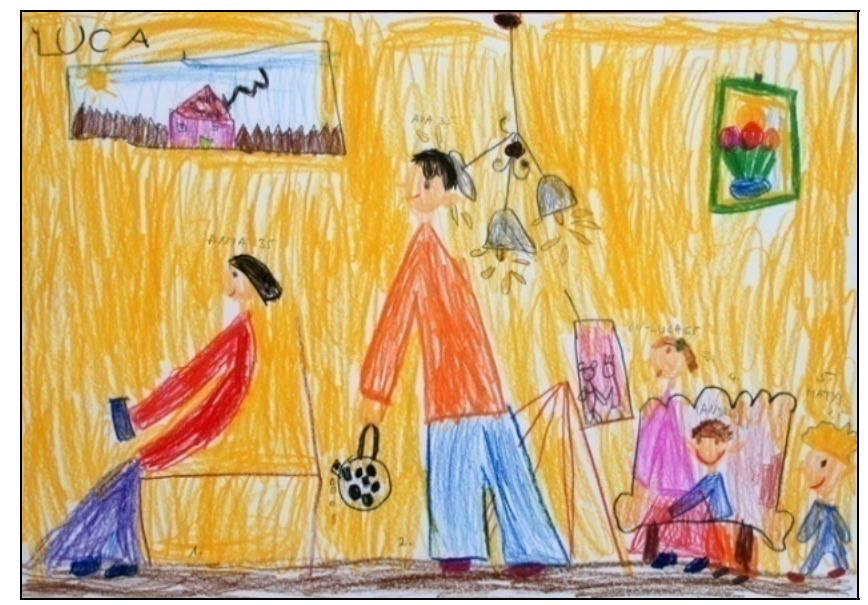

Figure 8. A 7-year-old girl, control group. The drawing is colourful, the members are in action, full coverage of paper surface. 
(2) Pattern 2 (see Figures 7 and 8):

Reaction time $>20$ seconds; low coverage of the surface (<30\%); lack of auxiliary elements; passive or moderate dynamics; lack of essential details, (e.g., mother's legs); use of eraser; their own figure is left out; few colors; high value on CBCL scale of "anxious/depressed" $(M>57)$.

(3) Pattern 3 (see Figures 9 and 10):

Moderate dynamics at the most; coverage of the paper surface $<50 \%$; size of their own figure $<25 \mathrm{~mm}$; average of figures $<35 \mathrm{~mm}$; low intensity of colors; Encapsulation/compartmentalization; high value on CBCL YSR scale of shyness and internalization $(M>59)$.

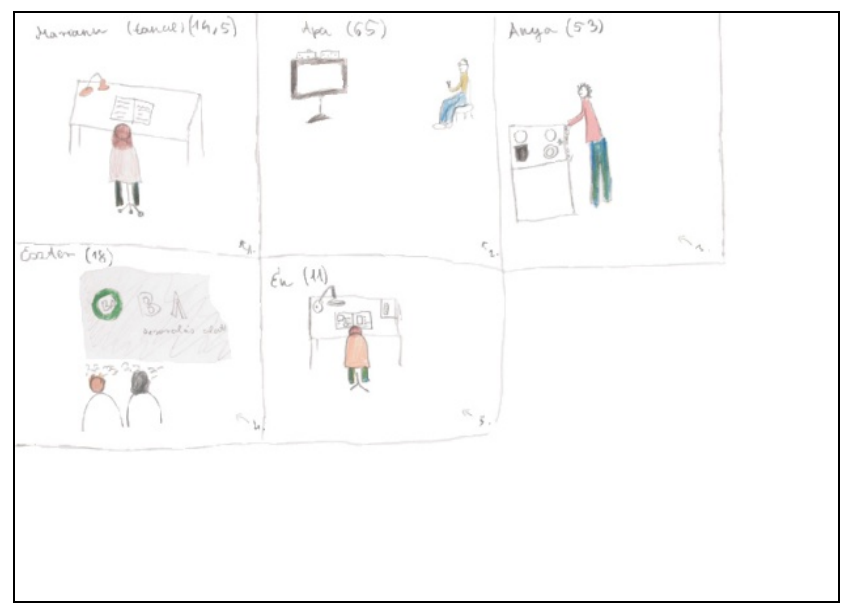

Figure 9. A 11-year-old girl, her sister suffers from tumor. The figure is encapsulated, separated from each other. The drawer (left side above) is looking like than her ill sister (right side down).

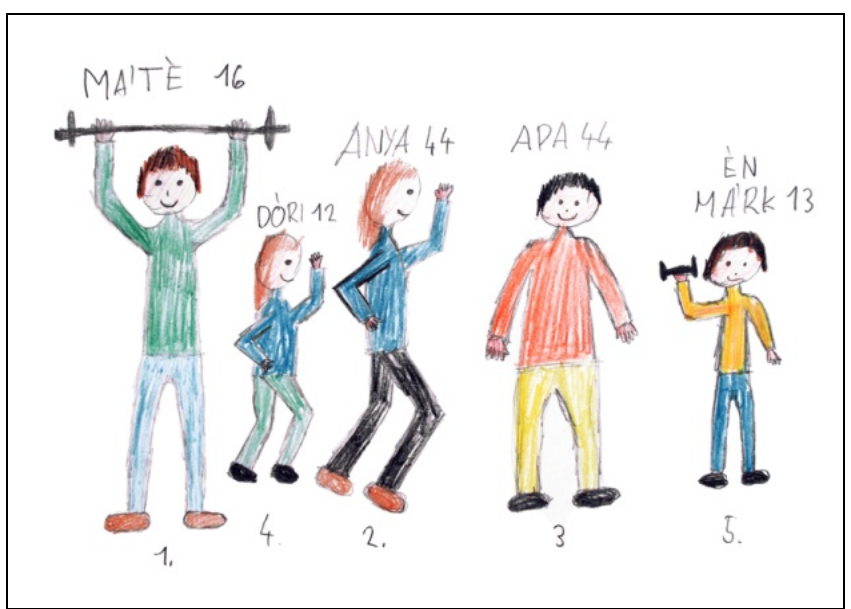

Figure 10. A 13-year-old boy, control group. Colourful (and bright), playful drawing. The members are in action. The effect of drawing is positive.

\section{Discussion}

The importance of the relationship between brothers and sisters is unquestionable, it is a force that shapes their personality. This impact is even higher when one of the brothers or sisters is struggling with a malign cancer. The results of this research are consistent with earlier results (Horwitz \& Kazak, 1990; Sahler et al., 1994; Alderfer et al., 2003; Houtzager et al., 2004), when we found increased problems of internalization and 
externalization in comparison to the control group. At the same time, we received new results which might bring us closer to a deeper understanding of this complex process, with a special respect to the projective drawing analysis that we have used.

The analyzed persons showed differences in the field of their behavioral, emotional and social relations. They indicated that the changes in the family caused difficulties for them, however, their extent did not reach a pathological level. The psychic phenomena found with the brothers and sisters of sick children showed a great individual variation, however, it seemed to be outlined that the strength and the nature of these symptoms were obviously influenced by age and the time passed since the diagnosis.

During the illness, the healthy brothers and sisters also witnessed the physical and psychic suffering of the child who received medical treatment, as well as the stressed condition of their parents. The consequence of these two factors is increased anxiety and isolation (both within the family and their age-group). With growing in age, the healthy brothers and sisters understood more and more at a cognitive level from the gravity of the illness, therefore, the worry about their brothers/sisters was increasing. At the same time, they also worried about themselves of suffering similar impacts. The condition of the brother/sister (altered body image, pain and physical weakness) undermines the belief of healthy brothers and sisters that only old people may suffer from a grave illness and life may only end in old age. This "law", which provides safety for the children, is replaced by the experience that anything bad may happen to anybody, further increasing the sense of uncertainty which characterises adolescence.

If the healthy brother/sister of the cancerous child is younger than 11 years, among the causes of the above described psychic phenomena, the inaccessibility of the mother may receive more emphasis than the actual cause (she stays with the sick brother/sister at the hospital), that is the reason why she is out of reach. In most families, the father undertakes extra work because of the increasing financial burden and it is not too rare that he often travels $100 \mathrm{~km}$ between the hospital and their home. Therefore, it is also difficult for the healthy children to reach the father. In case of younger children (7 to 11 years), everyday life is impossible without the presence of an adult. In the absence of parents, the tasks are often delegated to an elder brother/sister (if there is one). In these cases, we met two reactions from the elder brother/sister at the same time: aggression in a latent or manifested way, perhaps deviant behavior, while the level of anxiety was increasing because of the higher expectations, the worry about the sick brother/sister and the inaccessibility to the mother. Because of their age, adolescents desire independence and more autonomy, but they suddenly face increasing tasks and higher responsibility which may bring about anger and the multiplication of externalization symptoms.

From about 12 years of age, children on their own were able to manage daily routine tasks if necessary, however, they paid a high price for this: The rate of their isolation was increasing, according to our findings. If they cannot share their experience with their mother, especially in unknown and unusual situations which are full of fear, that would increase the level of anxiety. It is easier getting on at a practical level without help. However, they need their parents, especially their mother in order to digest their experience (especially the heavy ones) at this age.

According to our results, we have found a higher level of anxiety at the initial period immediately following the diagnosis of the illness, than one year later. It is because of the homeostasis is overturned: conditions changed overnight, requiring the system to be reorganized. Until a new family balance develops, the consequences of the overturned equilibrium are found besides the impact, producing anxiety by the diagnosis of tumor. As a new and somewhat stable family functioning developed, the level of anxiety seemed to be falling, 
however, it did not return to the control group's level even one year later.

The healthy brother/sister is isolated within the family in the age group and social relations to a certain extent. They are left alone with their experience, with their emotional tension, their fears and fantasies. "Once I tried to tell it to my best friend, but I gave up because I saw he did not even know what I was talking about. Next to him, I felt like somebody falling from Mars”, said a 15-year-old boy, whose younger brother suffers from leukemia. Individuals strive to share their world of experience, thus, establishing a relationship between the personal (internal) and the social world. Bruner (1989, p. 81) said that "One important reason of the fact that we tell stories is exactly to give a sense to those things we meet during our life". Language supports us in reducing the uncertainty deriving from certain situations and in giving expression to emotionally overloading events. If the mother is out of reach and it is also impossible to share the experience in the age group, language cannot help, either.

This idea takes us over to the other field of our research, the world of projective drawings. KFD, as we have explained above, clearly demonstrate that experience which is difficult to express with words will become accessible at image and graphical level, bridging the gap between the internal world of individual experience and the environment.

The common feature of the patterns that we have introduced is a prolonged reaction time. In times of serious family problems, children start doing their task after a longer hesitation, and family drawing is a difficult task during these times, because there is something which is not good to "talk" about. It has a paralyzing effect, it reduces creativity and in this way it increases the time necessary to start drawing (e.g., "How shall I draw my younger sister? She has no hair now, but normally she has"/“Poor buddy, shall I draw him with legs? It has been cut off and he will get a new one but it is not ready yet!”).

The other common feature is a low coverage of the paper surface. According to the projective interpretation, the drawing paper symbolizes the environment, and the spatial arrangement allows us to make a conclusion about the relationship between the designer and the environment (Buck, 1992). The high level of anxiety which goes together with the low coverage of the paper surface indicates that the environment is increasingly emphasized in contrast to the self for the brothers and sisters of children with cancer. If it is associated with encapsulation, as in patterns 1 and 3, it means an increased social withdrawal. The later phenomenon allows several interpretations: on the one hand, it may stand for the experience of exclusion from the family, but on the other hand, it may also indicate a deliberate demarcation from family events, tensions and the lack of open communication as well. The linear demarcation from the other members of the family concerns two figures most often: either the designer him/herself or the sick brother/sister is secluded, thus, indicating the emotional problems with him/her, isolation from him/her, inaccessibility, where fear may be in the background, just as real physical distance (hospital—home), or the two together.

Among the drawing phenomena that we have experienced, we also highlight the omission of the designer's own figure which is frequent in the target group. A similar, although undoubtedly not equally strong mark is the lack of essential details (e.g., arms and legs). The omission of the designer's own figure from the family drawing allows us to make a conclusion on emotional problems and those of self-appreciation, a negative self-image, as well as the prophetical position of the designer within the family.

Patterns 2 and 3 indicated a moderate dynamics at the most. While the control group depicted lively family events in motion, the target group often made static and stiff drawings, reflecting the real lethargy within the family of tumors children. 
The patterns shown are characterized by a moderate use of colors: The drawings have been made with few colours and even those are dim ("pale"), and there are no or only few motives enriching the pictures (auxiliary details). This kind of coexistence with these three variables is in sharp contrast to the results of the control group. Gregorian Azarian, DeMaria, and McDonald (1996) concluded in their studies that this phenomenon is found on the drawings of anxious children or of those struggling with neurotic symptoms.

\section{Conclusions}

Our results proved that the family drawings made by the healthy brothers and sisters of children suffering from a malign tumor carry the marks of changes within the family. With the help of these drawings, we got a more sophisticated picture about the internal conflicts of the children, their thinking and internal changes.

The altered dynamics of the family, the increased emotional tension and their everyday uncertainty indicated, with the help of drawing patterns shown above, that the KFD is suitable to uncover the children's deeper problems that are impossible or difficult to express verbally. Family drawings and other drawing techniques have also been used in case of children who had suffered physical or sexual abuse. These events of life are much more extreme than experiencing the grave illness of a brother/sister. KFD is a "sophisticated" procedure according to our results, suitable to indicate the consequences of "quieter" events of life, too.

At the same time, there are still many details to be clarified. Certain patterns may be clearly identified, indeed. However, we should analyze a higher number of elements and perhaps compare them with similar results of other target groups in order to give a rather sophisticated and well differentiated interpretation of co-occurrence. It does not mean the validity question of the test, but going one step deeper, but it is about the validation of patterns and the increasingly complex interpretation of the psychological content, expressed by these patterns.

We think that this kind of work is important because it helps us to understand deeper and in a more sophisticated way a non-normative event of life taking place in a family, therefore, contributing to the elaboration of more effective possibilities of intervention.

\section{References}

Achenbach, T. M. (1991a). Manual for child behaviour checklist/4-18 and 1991 profile. Department of Psychiatry, University of Vermont: Burlington, V. T..

Achenbach, T. M. (1991b). Manual for youth self report and 1991 profile. Department of Psychiatry, University of Vermont: Burlington, V. T..

Alderfer, M. A., Labay, L. E., \& Kazak, A. E. (2003). Brief report: Does posttraumatic stress apply to siblings of childhood cancer survivors? Journal of Pediatric Psychology, 28, 281-286.

Bendor, S. J. (1990). Anxiety and isolation in siblings of pediatric cancer patients: The need for prevention. Social Work in Health Care, 14, 17-35.

Bruner, J., \& Lucariello, J. (1989). Monologue as narrative recreation of the world. In K. Nelson (Ed.), Narrative form the crib. (pp. 73-97). Harvard University Press.

Buck, J. N. (1992). House-tree-person projective drawing technique manual and interpretive guide. Los Angeles: Western Psychological Services.

Burns, R. C., \& Kaufman, S. H. (1972). Action styles and symbols in kinetic family drawing (KFD). New York: Bruuner/Mazel.

Gregorian, V., Azarian, A., DeMaria, M., \& McDonald, L. (1996). Colors of disasters: The psychology of the "black sun”. Arts in Psychoteraphy, 23, 1-14.

Havermans, T., \& Eiser, C. (1994). Siblings of a child with cancer. Child: Care, Health and Development, 20(5), 309-322.

Horwitz, W. A., \& Kazak, A. E. (1990). Family adaptation to childhood cancer: Sibling and family system variables. Journal of Clinical Child Psychology, 19, 221-228. 
Houtzager, B. A., Grootenhuis, M. A., \& Last, B. F. (2001). Supportive groups for siblings of pediatric oncology patients: Impact on anxiety. Psycho-Oncology, 10, 315-324.

Houtzager, B. A., Grootenhuisa, M. A., Caron, H. N., \& Lasta, B. F. (2004). Quality of life and psychological adaptation in siblings of paediatric cancer patients, 2 years after diagnosis. Psycho-Oncology, 13, 499-511.

Labay, L. E., \& Walco, G. A. (2004). Brief report: Empathy and psychological adjustment in siblings of children with cancer. Journal of Pediatric Psychology, 29(4), 309-314.

Sahler, O. J., Roghmann, K. J., Carpenter, P. J., Mulhern, R. K., Dolgin, M. J., ..., Zeltzer, L. K. (1994). Sibling adaptation to childhood cancer collaborative study: Prevalence of sibling distress and definition of adaptation levels. Journal of developmental and Behavioural Pediatrics, 15(5), 353-366.

Vass, Z. (2001). Clinical application of kinetic family drawings. Hungarian Journal of Psychology, 56, 107-135.

Woodgate, R. L. (2006). Siblings' experiences with childhood cancer: A different way of being in the family. Cancer Nursing, 29, 406-414. 